\title{
Guia para estudos de revisão sistemática: uma opção metodológica para as Ciências do Movimento Humano
}

\author{
Isabelle Sena Gomes* \\ Iraquitan de Oliveira Caminha*
}

\begin{abstract}
RESUMO: Reunir conhecimento é um dos objetivos da consulta à literatura. Contudo, esta não está restrita a este fim. Objetivouse neste ensaio construir orientações práticas, cujo fio condutor pode ser seguido por diversas áreas de conhecimento, resultando em estudos com características de repetibilidade e reprodutibilidade. Uma revisão sistemática requer uma questão clara, critérios bem definidos e uma conclusão que forneça novas informações com base no conteúdo garimpado. Assim, revisões bem estruturadas podem auxiliar na atualização e construção de novas diretrizes para atuação profissional ou ida a campo em busca de soluções para artigos originais.
\end{abstract}

Palavras-chave: Revisão. Métodos. Domínios científicos. Literatura.

\section{INTRODUÇÃo}

Realizar novas pesquisas - seja no âmbito dos estudos de campo, experimentais, epidemiológicos ou qualquer outra modalidade - é fundamental para o avanço de qualquer vertente científica. No

\footnotetext{
"Programa Associado de Pós-Graduação em Educação Física (UFPB/UPE). João Pessoa, PB, Brasil. E-mail: euisabelle@yahoo.com.br

"Departamento de Educação Física. Programa Associado de Pós-graduação em Educação Física da Universidade Estadual de Pernambuco/Universidade Federal da Paraíba e do Programa de Pós-Graduação em Filosofia da Universidade Federal da Paraíba. João Pessoa, PB, Brasil. E-mail:caminhairaquitan@gmail.com
} 
entanto, há que se observar que todo e qualquer caminho percorrido pela ciência é permeado pelo aporte da revisão literária, sendo esta a base estruturante para a construção de questões norteadoras ou hipóteses (THOMAS; NELSON; SILVERMAN, 2012). Há alguns anos, diante de quadros de escassas possibilidades, buscava-se ampliação dos horizontes em estudos de caráter primário, atualmente, com a gama de recursos e avanços no âmbito acadêmico, a quantidade de estudos primários desenvolvidos permite que novos direcionamentos - inclusive a Prática Baseada em Evidência (PBE) ${ }^{1}$ - sejam vislumbrados a partir de estudos de revisão sistemática (WRIGHT et al, 2007).

A revisão de literatura (ou revisão narrativa) é sempre recomendada para o levantamento da produção científica disponível e para a (re)construção de redes de pensamentos e conceitos, que articulam saberes de diversas fontes na tentativa de trilhar caminhos na direção daquilo que se deseja conhecer. No entanto, este método, de caráter descritivo-discursivo, não costuma apresentar características de reprodutibilidade e repetibilidade, tornando-se demasiadamente empírico, obscuro, e/ou inconclusivo na opinião de alguns pesquisadores (SEGURA-MUÑOZ et al., 2002). Esta afirmativa não nega a importância das revisões narrativas, mas, chama atenção para novas possibilidades ainda pouco utilizadas apesar de sua viabilidade. Isto porque, nos últimos 10 anos novas formas de análise criadas e utilizadas pelas Ciências da Saúde vem permitindo a elaboração de estudos de síntese, que constituem por si mesmos e por seus métodos bem definidos - pesquisas, e não apenas levantamento da literatura disponível (MEDINA; PAILAQUILÉN, 2010).

Pensando nisso, a revisão sistemática vem sendo utilizada como método para suprir a lacuna da inconclusão deixada pelas revisões narrativas. "Os pesquisadores precisam das Revisão Sistemática

\footnotetext{
${ }^{1} \mathrm{~A}$ PBE surgiu no Canadá, por volta dos anos 80 , com o intuito de aprimorar a prática clínica e a qualidade do ensino, sendo conhecida inicialmente por Medicina Baseada em Evidencia, e posteriormente incorporada por outros campos (DE-LA-TORRE-UGARTE-GUANILO;TAKAHASHI; BERTOLOZZI, 2011).
} 
Guia para estudos de revisão sistemática: uma opção...

(RS) para resumir os dados existentes, refinar hipóteses, estimar tamanhos de amostra e ajudar a definir agendas de trabalho futuro considerados como seus sujeitos" (MEDINA; PAILAQUILÉN, 2010, p. 7). Por este motivo, o presente ensaio assinala a revisão sistemática (ou síntese criteriosa) como opção para não apenas acastelar informações, mas acompanhar o curso científico de um período específico, chegando ao seu ápice na descoberta de lacunas e direcionamentos viáveis para a elucidação de temas pertinentes. Objetivou-se então, construir orientações práticas, cujo fio condutor pode ser seguido por diversas áreas do conhecimento, resultando em estudos com características de repetibilidade e reprodutibilidade.

De acordo com Riera, Abreu e Ciconelli (2013), a primeira metanálise foi publicada em 1904, mas foi no final dos anos 80 que as revisões sistemáticas e metanálises se destacaram na área da saúde. Por volta de 1992, o termo "meta-analysis" foi incluído entre os Descritores das Ciências da Saúde, permitindo que revisões sistemáticas sejam rapidamente encontradas em bases de dados nacionais e internacionais.

Uma revisão sistemática requer, como qualquer estudo, uma questão clara, critérios de seleção bem definidos - de modo que garanta a qualidade dos estudos sintetizados e possa ser reproduzida por outrem - e uma conclusão que forneça novas informações com base no conteúdo garimpado (THOMAS; NELSON; SILVERMAN, 2012). Assim, revisões bem estruturadas podem auxiliar na atualização e construção de novas diretrizes para atuação profissional ou ida a campo em busca de soluções para artigos originais.

No decorrer deste ensaio, será feita uma descrição pragmática das etapas que constituem o processo de elaboração de um estudo de revisão sistemática, à luz da literatura disponível. Em cada passo, um breve detalhamento será apresentado com o intuito de aproximar pesquisadores deste método ainda pouco explorado em algumas áreas de conhecimento. Por fim, um esquema reunirá o que foi encontrado e considerado mais viável na literatura, com o objetivo de elucidar e esmiuçar a proposta desta pesquisa. 


\section{MAS AFINAL, O QUE É UMA REVISÃo SISTEMÁTICA?}

O método de revisão sistemática da literatura consiste em um movimento que tem base em critérios pré-determinados e evidências científicas consistentes, tendo como fim colaborar com a escolha de estudos e/ou ferramentas para o desenvolvimento de artigos com informações originais (SCHÜTZ; SANT'ANA; SANTOS, 2011). "Métodos sistemáticos são usados para evitar viés e possibilitar uma análise mais objetiva dos resultados, facilitando uma síntese conclusiva" (SAMPAIO; MANCINI, 2007, p. 84). Nas palavras de De-La-Torre-Ugarte-Guanilo, Takahashi e Bertolozzi (2011, p. 1261) é "uma metodologia rigorosa proposta para: identificar os estudos sobre um tema em questão, aplicando métodos explícitos e sistematizados de busca; avaliar a qualidade e validade desses estudos, assim como sua aplicabilidade". Com base nesta premissa, a revisão sistemática figura como método útil - embora ainda pouco explorado - para as ciências do movimento humano, oferecendo capacidade de síntese e novos direcionamentos.

A Revisão Sistemática pode estar ancorada em pesquisas qualitativas ou quantitativas, em suma, isso dependerá do objetivo e pergunta de pesquisa. Os resultados podem ser expostos na forma de conclusão, análise ou síntese. Contudo, na RS qualitativa devem estar asseguradas: a validade descritiva (identificação de estudos relevantes), interpretativa (correspondência entre o registrado pelo revisor e o conteúdo do estudo), teórica (credibilidade dos métodos desenvolvidos) e pragmática (aplicabilidade do conhecimento gerado). (DE-LA-TORRE-UGARTE-GUANILO; TAKAHASHI; BERTOLOZZI, 2011).

Diversos autores classificam de formas diferentes revisões sistemáticas que possuem abordagem qualitativa, quantitativa, ou atendendo a ambos os métodos, sendo respectivamente chamadas de: síntese com análise estatística (podendo esta dividir-se em revisão descritiva ou metanálise), síntese (com uma variedade de 
Guia para estudos de revisão sistemática: uma opção...

possibilidades, devido à riqueza de detalhes própria das pesquisas qualitativas) e revisão integrativa (que une de alguma forma os métodos já citados) (DE-LA-TORRE-UGARTE-GUANILO; TAKAHASHI; BERTOLOZZI, 2011). A figura 1 resume as possibilidades metodológicas da revisão sistemática. O quadro 1 traz alguns dos tipos de revisões sistemáticas. Estas foram realizadas nos anos de 2012 e 2013 e coletadas em: 18 de novembro de 2013 em revistas brasileiras de Educação Física, com o intuito de exemplificar e explicitar a relação entre objetivos, bases de dados selecionadas e apresentação dos principais achados.

Figura 1 - Possibilidades metodológicas da revisão sistemática

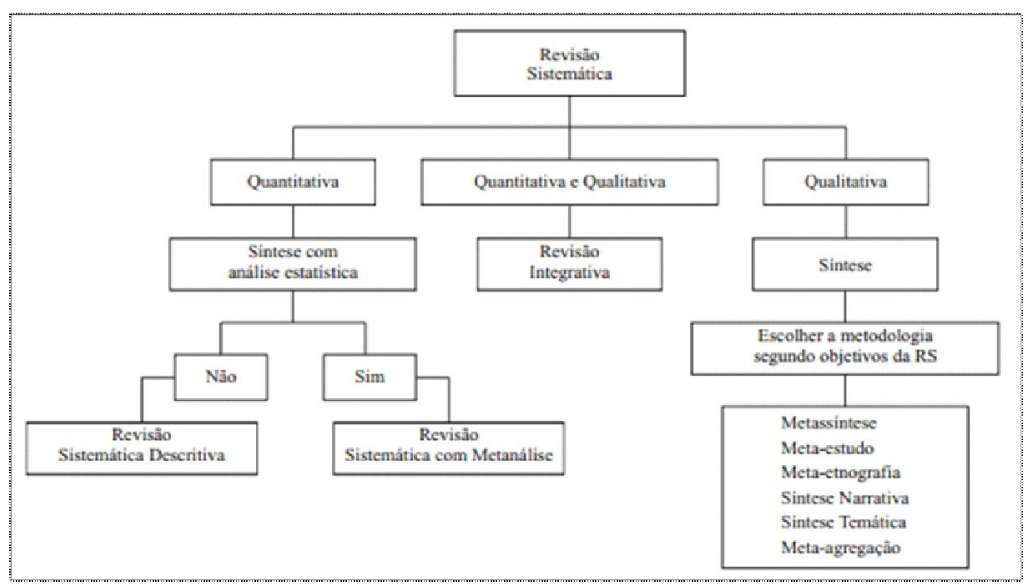

Fonte: De-La-Torre-Ugarte-Guanilo, Takahashi e Bertolozzi, 2011, p. 1259. 
Quadro 1 - Exemplos de Revisão Sistemática: Qualitativa (RSQ); Integrativa (RSI) e Quantitativa - Meta-análise (RSQT)

\begin{tabular}{|c|c|c|c|c|c|}
\hline $\begin{array}{l}\text { Autores/ } \\
\text { tipo }\end{array}$ & Objetivo & $\begin{array}{l}\text { Bases } \\
\text { de } \\
\text { dados }\end{array}$ & $\begin{array}{c}\text { Descritores } \\
\text { utilizados }\end{array}$ & Critérios de inclusāo & Achados \\
\hline $\begin{array}{l}\text { Noll, } \\
\text { Tarragô } \\
\text { Candotti e } \\
\text { Vieira } \\
\text { (2012). } \\
\text { (RSQ) }\end{array}$ & $\begin{array}{l}\text { Apresentar e } \\
\text { discutir os } \\
\text { programas de } \\
\text { Escola Postural } \\
\text { desenvolvidos para } \\
\text { escolares } \\
\text { no Brasil. }\end{array}$ & $\begin{array}{l}\text { Scopus, } \\
\text { Science } \\
\text { Direct, } \\
\text { PubMed, } \\
\text { Scielo } \\
\text { Banco de } \\
\text { Teses e } \\
\text { Dissertaçõe } \\
\text { S da CAPES }\end{array}$ & $\begin{array}{l}\text { Postural } \\
\text { Program, } \\
\text { Postural } \\
\text { School, Back } \\
\text { School, Back } \\
\text { Care } \\
\text { Education, } \\
\text { Back } \\
\text { Education, e } \\
\text { os mesmos } \\
\text { termos em } \\
\text { português. }\end{array}$ & $\begin{array}{l}\text { Desenvolver um programa } \\
\text { teórico e/ou prático } \\
\text { educativo e/ou preventivo } \\
\text { relacionado ao ensino da } \\
\text { execução adequada das } \\
\text { AVD's e assuntos } \\
\text { relacionados à postura } \\
\text { corporal e coluna vert ebral. } \\
\text { Datar de } 1970 \text { em diante, } \\
\text { sernacional, ter populaçăo } \\
\text { alvo: crianças e/ou } \\
\text { adoles centes. } \\
\text { (Selecionados: } 291 \text { artigos. } \\
\text { Incluídos:9) }\end{array}$ & $\begin{array}{l}\text { Os programas de Escola } \\
\text { Postural contribuem para a } \\
\text { aquisição de conhecimentos } \\
\text { teóricos e de hábitos posturais } \\
\text { saudáveis durante a execução } \\
\text { das atividades de Vida Diárias. }\end{array}$ \\
\hline $\begin{array}{l}\text { Silvestrin et } \\
\text { al. (2013) } \\
\text { (RSQT) }\end{array}$ & $\begin{array}{l}\text { Analisar a } \\
\text { associação entre } \\
\text { grau de } \\
\text { escolaridade } \\
\text { materna e peso de } \\
\text { nascimento, } \\
\text { considerando-se a } \\
\text { hipótese de que a } \\
\text { utilização em } \\
\text { excesso das } \\
\text { tecnologias na área } \\
\text { da saúde, assim } \\
\text { como a escassez } \\
\text { de recursos, pode } \\
\text { produzir desfechos } \\
\text { similares. }\end{array}$ & MEDLINE & $\begin{array}{l}\text { Socio- } \\
\text { economic } \\
\text { Factors, } \\
\text { Infant, } \\
\text { Low Birth } \\
\text { Weight, } \\
\text { Cohort } \\
\text { Studies, } \\
\text { Cross- } \\
\text { Sectional } \\
\text { Studies. }\end{array}$ & $\begin{array}{l}\text { Delineamento de estudo } \\
\text { transversal ou de co orte; } \\
\text { publicação na língua } \\
\text { inglesa, portuguesa ou } \\
\text { es panhola; o baixo peso } \\
\text { ao nascer (<2.500 g) } \\
\text { como desfecho e a } \\
\text { variável grau de } \\
\text { es colaridade mat erna } \\
\text { distribuída em três estratos } \\
\text { (baixo, médio e alto) } \\
\text { (Selecionados: } 729 \text { artigos. } \\
\text { Incluídos: } 9 \text { ) }\end{array}$ & $\begin{array}{l}\text { A meta-análise não comprovou } \\
\text { a hipótese proposta. Identificou- } \\
\text { se um efeito protetor de } 33 \% \\
\text { para o risco de BPN entre as } \\
\text { mulheres com escolaridade } \\
\text { elevada, quando comparado } \\
\text { com a categoria de baixa } \\
\text { educação materna. } \\
\text { Diferentemente, ao avaliar o } \\
\text { risco de baixo peso em relação } \\
\text { às mães de escolaridade média, } \\
\text { quando comparadas às de } \\
\text { escolaridade baixa, não houve } \\
\text { um resultado significativo. }\end{array}$ \\
\hline $\begin{array}{l}\text { Silva et al } \\
(2013) \\
(\mathrm{RSI})\end{array}$ & $\begin{array}{l}\text { Revisar os fatores } \\
\text { que estimulam } \\
\text { a utilização dos } \\
\text { espaços públicos de } \\
\text { lazer e direcionar os } \\
\text { gestores públicos } \\
\text { para que os } \\
\text { indivíduos tenham } \\
\text { melhor qualidade de } \\
\text { vida. }\end{array}$ & $\begin{array}{l}\text { PubMed, } \\
\text { LILACS } \\
\text { SciELO, } \\
\text { (Disp.no } \\
\text { portal } \\
\text { CAPES) }\end{array}$ & $\begin{array}{l}\text { Parques } \\
\text { urbanos, } \\
\text { Praças, } \\
\text { Parques } \\
\text { públicos, } \\
\text { Áreas verdes, } \\
\text { Square e } \\
\text { Urban park. }\end{array}$ & $\begin{array}{l}\text { Artigos originais } \\
\text { relacionados aos espaços } \\
\text { públicos de lazer, } \\
\text { publicados no período de } \\
2005 \text { a } 2010 \text {, em } \\
\text { periódicos nacionais e } \\
\text { internacionais, nos idiomas } \\
\text { português e inglês, e } \\
\text { pesquisas realizadas com } \\
\text { seres humanos } \\
\text { (Selecionados: } 9.052 \\
\text { artigos. Incluídos: } 11 \text { ). }\end{array}$ & $\begin{array}{l}\text { Predominam estudos sobre } \\
\text { lazer nas regiões Sul e Sudeste, } \\
\text { comprometendo a análise da } \\
\text { realidade dos espaços de lazer, } \\
\text { benefícios e } \\
\text { fragilidades encontradas nas } \\
\text { demais regiões. }\end{array}$ \\
\hline $\begin{array}{l}\text { Vasconcelos } \\
\text { etal (2013) } \\
\text { (RSI) }\end{array}$ & $\begin{array}{l}\text { Analisar a eficácia } \\
\text { de intervençōes que } \\
\text { utilizam o telefone } \\
\text { como estratégia } \\
\text { para o controle } \\
\text { glicêmico de adultos } \\
\text { portadores de } \\
\text { Diabetes Mellitus } \\
\text { tipo } 2 \text {. }\end{array}$ & $\begin{array}{l}\text { Cochrane } \\
\text { PubMed/ } \\
\text { MEDLINE/ } \\
\text { LILACS } \\
\text { CINAHL }\end{array}$ & $\begin{array}{l}\text { Diabet es } \\
\text { Melitus, } \\
\text { Diabet es } \\
\text { Melitus, Type } \\
2, \\
\text { Telephone, } \\
\text { Intervent ion } \\
\text { Studies; e os } \\
\text { mesmos } \\
\text { termos em } \\
\text { português. }\end{array}$ & $\begin{array}{l}\text { Artigos científicos que } \\
\text { atendessem à questão } \\
\text { norteadora, escritos nos } \\
\text { idiomas inglês, português } \\
\text { e espanhol. disponíveis na } \\
\text { íntegra online. } \\
\text { (Selecionados: } 280 \text { artigos. } \\
\text { Incluídos: } 9 \text { ). }\end{array}$ & $\begin{array}{l}\text { Houve redução, muitas vezes } \\
\text { significativa dos níveis de } \\
\text { hemoglobina glicada e da } \\
\text { glicemia venosa de jejum nos } \\
\text { pacientes que estavam } \\
\text { incluídos no grupo de } \\
\text { intervenção, per mitindo a } \\
\text { conclusão de que as } \\
\text { intervenções que utilizam o } \\
\text { telefone como estratégia são } \\
\text { eficazes no controle glicêmico } \\
\text { dos pacientes que possuem } \\
\text { DM2. }\end{array}$ \\
\hline
\end{tabular}

Fonte: Dos Autores

Sobre as subdivisões da revisão sistemática, Souza, Silva e Cavalho (2010) escrevem que meta-análise é um método de revisão que une evidências de estudos unicamente primários a partir de instrumentos estatísticos, aumentando a objetividade e a validade dos resultados. Cabe ressaltar que a conclusão mais importante de uma meta-análise é o resumo quantitativo de resultados. "Na meta- 
Guia para estudos de revisão sistemática: uma opção...

análise, é essencial que as diferenças estatísticas dos resultados dos estudos sejam pesquisadas", com olhar acurado sobre homogeneidades e heterogeneidades encontradas (MEDINA; PAILAQUILÉN, 2010, p. 6). As varíaveis envolvidas e levadas em conta pelo estudo, o tipo de amostra, procedimento, resultados obtidos, etc.; são sintetizados estatisticamente e/ou comparados no decorrer do estudo, objetivando uma conclusão à luz de estudos primários.

Enquanto nas pesquisas clínicas e quantitativas foram desenvolvidos estudos que utilizaram da meta-análise para sínterese de evidência e elaboração de protocolos e condutas à partir da literatura, no campo das pesquisas qualitativas novas vertentes metodológicas também foram desenhadas. A revisão sistemática qualitativa, é uma síntese rigorosa de pesquisas relacionadas à questão norte do estudo (frequente em randomizações), envolvendo também a interpretação dos dados organizados. Quando as revisões agrupam resultados de outros estudos, porém não aplicam análise estatística de modo global, são chamadas de RS qualitativas. O objetivo principal destas é levar em conta as similaridades e diferenças importantes entre as pesquisas já realizadas, no sentido de ampliar as possibilidades interpretativas dos resultados, construindo (re) leituras ampliadas (HOEFELMANN; SANTOS; MORETTI-PIRES, 2012).

Alguns autores, como De-La-Torre-Ugarte-Guanilo, Takahashi e Bertolozzi (2011), subdividem a RS qualitativa em outros tipos específicos, diferindo-os pela priorização de um ou outro aspecto (Figura 1). Em contrapartida, Medina e Pailaquilén (2010) e Hoefelmann, Santos e Moretti-Pires (2012) consideram apenas a existência da meta-síntese (sendo as demais nomenclaturas variações desta) em oposição à metanálise, escrevendo que ambas não podem estar presentes no mesmo estudo.

Apresentando-se como uma possibilidade de flexibilização, a revisão integrativa é mais ampla por se tratar de uma junção de métodos. Ela permite a inclusão de estudos experimentais e nãoexperimentais e combina um vasto leque de propósitos com perspectivas teóricas, gerando a possibilidade de olhar um mesmo fenômeno de diferentes perspectivas. 


\section{DELINEAMENTO PARA A CONSTRUÇÃo dE UMA REVISÃo SISTEMÁTICA}

A construção prévia do arcabouço metodológico colabora para que a pesquisa tenha a confiabilidade aumentada, reduzindo a ocorrência de vieses. Além disso, a falta de critérios bem definidos configura-se como um dos principais entraves para o desenvolvimento deste tipo de estudo, uma vez que a repetibilidade e reprodutibilidade são valorizadas, assim como em outros tipos de pesquisa.

Com o decurso dos anos, algumas instituições criaram e consolidaram sugestões próprias de construção de revisões sistemáticas, entre elas destaca-se - com base no que escreve Castro (2009) - o Instituto Cochrane (Cochrane Handbook) e o NHS (Centre for Reviews and Dissemination, University of York). Outros métodos como o PRISMA - antigo QUOROM - são utilizados também, porém, com mais frequência em estudos internacionais (MOHER et al., 2009), e por isso fogem ao escopo deste estudo. Busca-se neste estudo sugerir percursos metodológicos à luz destas duas propostas, que são complementares e amplamente aceitas no meio acadêmico. A realização de revisões de qualidade envolve no mínimo dois pesquisadores independentes e cabe frisar que os procedimentos que se seguem devem ser pré-estabelecidos em um projeto de pesquisa garantindo a qualidade do resultado.

O Instituto Cochrane estabelece sete passos para a realização da revisão sistemática, a saber: (1) Formulação da pergunta, (2) Localização e seleção dos estudos (3) Avaliação crítica dos estudos (4) Coleta de dados, (5) Análise e apresentação dos dados, (6) Interpretação dos dados e (7) Aprimoramento e atualização da revisão (HIGGINS; GREEN, 2011).

(1) Formulação da pergunta - O primeiro passo a ser dado no início de qualquer estudo é estabelecer o que se deseja pesquisar. Questões mal formuladas podem conduzir a decisões obscuras sobre o que incluir na revisão posteriormente.

(2) Localização e seleção dos estudos em bases de dados indexadas - Esta etapa realiza-se em bases de dados eletrônicas 
(databases) indexadas (a partir da seleção de unitermos, também conhecidos como descritores ou palavras-chave). Entre as bases de dados disponíveis, principalmente para construção de revisões de intervenção em saúde, a MEDLINE e a EMBASE são bastante utilizadas. Contudo, outras bases de dados são utilizadas com frequência, sendo muitas delas disponíveis gratuitamente e com conteúdo predominantemente online. O Centre For Reviews And Dissemination (2012) aponta em sua publicação online - direcionada a pesquisadores - alguns dos bancos de dados mais utilizados mundialmente. Com base neste documento, o quadro 2 sintetiza algumas das opções viáveis para a área das Ciências do Movimento Humano, bem como seu escopo e sítio eletrônico.

Quadro 2 - Bancos de dados, escopo e localização eletrônica propostos pelo CENTRE FOR REVIEWS AND DISSEMINATION (CRD) para realização de revisões sistemáticas.

\begin{tabular}{|c|c|c|}
\hline Banco de dad os & Escopo & Disponível em \\
\hline $\begin{array}{l}\text { Índice de Ciências } \\
\text { Sociais Aplicadas e } \\
\text { Res umos (AS SIA) }\end{array}$ & $\begin{array}{l}\text { Abrange a saúde, serviços sociais, psicologia, } \\
\text { sociologia, economia, política, relações raciais } \\
\text { e educação. }\end{array}$ & $\begin{array}{l}\text { http://www.csa. } \\
\text { com/factsheets/ } \\
\text { as sia-s et-c.php }\end{array}$ \\
\hline BIOSIS & $\begin{array}{l}\text { Grande banco de dados que cobre a literatura das } \\
\text { ciências da vida. Con tém artigos de periódicos e } \\
\text { reuniões da conferência, mas relatórios, livros e } \\
\text { patentes também es tão incluídos. }\end{array}$ & $\begin{array}{l}\text { http://thomsonr } \\
\text { euters.com/biosi } \\
\text { s-citation-index }\end{array}$ \\
\hline $\begin{array}{l}\text { Cochrane Database } \\
\text { of Systematic } \\
\text { Reviews (CDSR) }\end{array}$ & $\begin{array}{l}\text { É uma coleção de revis ões sistem áticas, regu larmen te } \\
\text { atuali zada, cobrindo cuidad os em saúde. Disponível } \\
\text { como parte da Biblioteca Cochrane. }\end{array}$ & $\begin{array}{l}\text { http://www.coc } \\
\text { hrane.org }\end{array}$ \\
\hline Literatura Latino- & Banco de dados de pesquisa em saúde da América & http://lilacs.bvs a \\
\hline $\begin{array}{l}\text { Americana e do } \\
\text { Caribe em Ciências } \\
\text { da Saúde (LILACS) }\end{array}$ & Latina e Caribe. Contém artigos de 670 revistas. & lud .org \\
\hline $\begin{array}{l}\text { National Library of } \\
\text { Medicine } \\
(\text { MEDLINE) }\end{array}$ & $\begin{array}{l}\text { Contém mais de } 18 \text { milhões de referências. Contém } \\
\text { in formações sobre biomedicina e saúde, ciências da } \\
\text { vida, ciências do comportamento, ciências químicas e } \\
\text { Bioengenharia. MEDLINE é o maior componente do } \\
\text { PubMed. }\end{array}$ & $\begin{array}{l}\frac{\text { http://www.ncbi }}{\text { nlm.nih.gov/sit }} \\
\text { es/entrez }\end{array}$ \\
\hline PsycINFO & $\begin{array}{l}\text { Abran ge a literatura de psicologia e áreas afins. Ele } \\
\text { inclui livros, liv ro capítulos e dissertações, bem como } \\
\text { artigos. }\end{array}$ & $\begin{array}{l}\text { http://www.apa. } \\
\text { org/pubs/databa } \\
\text { ses/psycinfo/ind } \\
\text { ex.aspx }\end{array}$ \\
\hline Scopus & $\begin{array}{l}\text { Banco de dados, resumos e citações, da literatura } \\
\text { revisad a por pares e fontes web de qualidade } \\
\text { com ampla cobert ura em todo o assunto científico, } \\
\text { técnico, literatura das ciências médicas e sociais. }\end{array}$ & $\begin{array}{l}\text { http://www.info } \\
\text { scivers e.com/sc } \\
\text { opus/about }\end{array}$ \\
\hline $\begin{array}{l}\text { Scientific Electronic } \\
\text { Library Online } \\
\text { (Scielo) }\end{array}$ & $\begin{array}{l}\text { Biblio teca eletrônica que abrange uma coleção } \\
\text { selecion ada de periódicos científicos brasileiros. } \\
\text { Possui uma grande varied ade de temas rel acionados à } \\
\text { Filoso fia, com artigos completos disponíveis para } \\
\text { download. }\end{array}$ & $\begin{array}{l}\text { http://www.scie } \\
\text { lo.org/index.ph } \\
\text { p?lang=pt }\end{array}$ \\
\hline
\end{tabular}

Fonte: Autores

Movimento, Porto Alegre, v. 20, n. 01, p. 395-411, jan/mar de 2014. 
Faz-se necessário o cuidado com a seleção das bases de dados, pois, as mesmas precisam corresponder às expectativas quanto à temática abordada, de modo que a opção pelas que possuem baixa probabilidade de dispor do conteúdo abordado poderá comprometer o processo de garimpar informações. Ao encontrar os estudos de interesse, o pesquisador poderá deparar-se com algumas limitações, pois, certos manuscritos não estão disponíveis na íntegra, requerendo contato com os portadores das informações.

(3) Avaliação crítica dos estudos - De posse de todos os estudos a serem incluídos, são estabelecidos critérios para determinar a sua validade e se há possibilidade dos resultados possuírem vieses. Depois desta avaliação crítica - que pode levar em conta informações como método, randomização, classificação do periódico no Webqualis , etc. - devem restar apenas os estudos confiáveis. Aqueles que forem excluídos podem ser citados no decorrer da pesquisa se for pertinente a divulgação da razão de sua exclusão. Ressalta-se a obrigatoriedade da exclusão de estudos de revisão.

(4) Coleta de dados - Durante este passo, que se configura, sobretudo, como um movimento organizacional, todas as variáveis contidas nos estudos devem ser levantadas e investigadas com atenção, além das características do método, suas limitações e o desfecho do estudo.

(5) Análise e apresentação dos dados - Com base nas semelhanças entre artigos, os dados serão agrupados para a obtenção das conclusões finais (ou da metanálise se este for o caso). Cada um destes agrupamentos deve - preferencialmente - ser préestabelecido previamente, evitando a tendenciosidade.

(6) Interpretação dos dados e (7) Aprimoramento e atualização da revisão - Nas etapas finais, a redação dos resultados deve ser feita levando-se em conta a questão norteadora estabelecida no primeiro passo supracitado. Assim, o estudo terá em seu término respondido àquilo que se propõe. Deve conter em sua redação como se desenrolaram todas as etapas anteriores, de modo que fique claro para o leitor como o estudo foi construído, e não apenas o resultado 
Guia para estudos de revisão sistemática: uma opção...

obtido. Um esforço final é feito na direção de manter a revisão sistemática atualizada de acordo com o surgimento de novas pesquisas sobre o tema.

Traçando um paralelo com o método proposto pelo instituto Cochrane, será exposta a seguir a forma organizacional sugerida pelo CDR Report (NHS). A descrição detalhada da trajetória para uma revisão sistemática segundo o NHS pode dividir-se em nove etapas, ou três estágios, a saber: Estágio 1: Planejamento e revisão; Estágio 2: Conduzindo a revisão; Estágio 3: apresentação do relatório e divulgação.

Estágio 1 - (a) Identificação da necessidade da revisão, (b) preparação de uma proposta para a revisão sistemática, (c) Desenvolvimento de um projeto de revisão.

Estágio 2 - (d) Identificação da literatura, (e) Seleção dos estudos, (f) Avaliação da qualidade dos estudos, (g) Extração dos dados e monitorização do progresso, (h) Síntese dos dados.

Estágio 3 - (i) Relatório e recomendações, transferindo evidências para a prática.

Para ilustrar do que se trata a elaboração de critérios para seleção dos estudos, cabe observar o estudo de natureza Integrativa de Gonçalves, Campana e Tavares (2012), cujos critérios de inclusão foram: (a) publicação dos últimos 30 anos, (b) estudos experimentais ou semi-experimentais, (c) estudos qualitativos ou quantitativos, (d) estudos com descrição da intervenção de atividade física realizada, (e) intervenção de no mínimo 12 semanas. Os critérios de exclusão adotados foram: artigos que (a) não realizaram intervenção ou o fizeram por menos de 12 semanas; (b) estudos que não descreveram as atividades, e (c) estudos que não usaram questionários validados em sua coleta de dados - quantitativos.

De forma sintética e pragmática, pode-se observar que ambos os métodos são em certo ponto equivalentes, de modo que o estágio 1 (NHS) aproxima-se do passo 1 (Cochrane), e assim sucessivamente nos demais estágios. Nesse contexto, é viável unir 
ambas as propostas visando obter uma terceira dotada de tanta clareza quanto possível, facilitando a familiarização dos que desejam realizar esta modalidade de pesquisa com a mesma.

Segura-Muñoz et al. (2002), Sampaio e Mancini (2007) complementam o pensamento proposto por Castro (2009) - de munirse de ambos os métodos - e acrescentam o que chamam de reuniões de consenso. AFigura 2 sintetiza esta proposta de união metodológica.

Figura 2 - Junção das propostas viáveis encontradas na literatura em um esquema-proposta de percurso

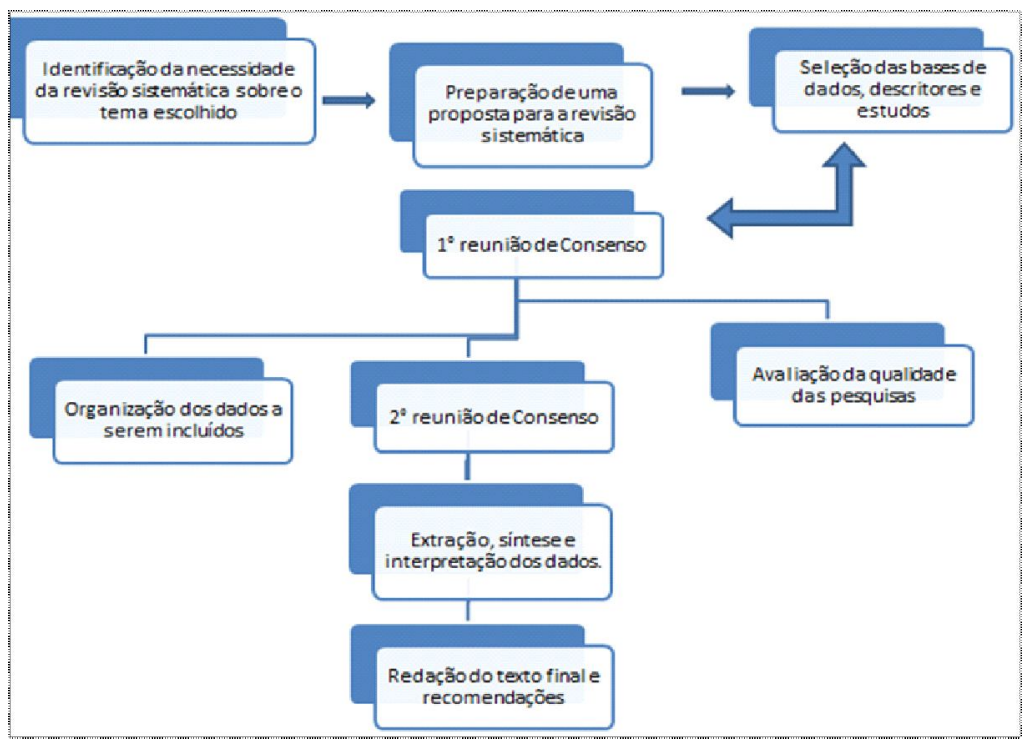

Fonte: Dos Autores

As reuniões de consenso são espaços de discussão para solucionar percalços que possam vir surgir, evitando vieses ou demasiada dissonância entre os realizadores da pesquisa (recomendam-se dois ou mais pesquisadores). Geralmente ocorrem depois da primeira seleção de estudos (passo 3 do método Cochrane; estágio 2 - NHS) e pode ocorrer novamente se houver necessidade. Nesta primeira reunião, espera-se que os estudos selecionados pelos autores sejam os mesmos. Isto porque os critérios de inclusão e 
Guia para estudos de revisão sistemática: uma opção...

exclusão devem proporcionar objetividade no método. Contudo, pode haver dúvida ou necessidade de discussão sobre a inclusão de algum(ns) artigo(s), e é este problema que a reunião de consenso deve sanar. Se o número de pesquisadores for par e a dúvida permanecer, um outro pesquisador pode ser consultado sem prejuízos ao método, com a finalidade de solucionar a questão.

\section{Considerações finaIS}

Compreender como se dá o desenvolvimento de uma revisão sistemática pode auxiliar o pesquisador na tarefa de construir pesquisas que são de fato importantes para os avanços científicos. $\mathrm{O}$ percurso que segue o realizador deste tipo de estudo é marcado pela necessidade de estabelecer critérios objetivos e consistentes para a seleção das informações que irão conduzir à solução da pergunta norteadora, caso isto não ocorra a síntese criteriosa está fadada à confusão de ideias e, portanto, de resultados. $\mathrm{O}$ objetivo final desse processo foi proporcionar ferramentas para melhorar a qualidade dos cuidados oferecidos pelos profissionais da saúde. Para tanto, foi elaborado um esquema que uniu as sugestões mais viáveis disponíveis na literatura, facilitando o entendimento. Apresentou-se como limitação para este método a impossibilidade de melhor detalhar cada tipo de pesquisa, uma vez que as três vertentes apresentadas (qualitativa, quantitativa e integrativa) carecem de atenção específica. Nessa conjuntura, fica a sugestão para futuros estudos que abordem com minúcia cada percurso metodológico. 
Guía de revisión sistemática de estudios: una opción para la metodología de las Ciencias del Movimiento Humano

Resumen: Reunir conocimiento es uno de los objetivos de la literatura consulta. Sin embargo, esto no se limita a este propósito. El objetivo de esta prueba de compilación orientación práctica, cuya rosca puede ser seguido por varias áreas de conocimiento, lo que resulta en estudios con características de repetibilidad y reproducibilidad. Una pregunta de la revisión sistemática requiere un criterio claros y bien definidos y una conclusión que proporciona nueva información basada en el contenido criticada. Por lo tanto, las revisiones bien estructuradas pueden ayudar en la mejora y construcción de nuevas directrices para el ejercicio profesional o ir al campo en busca de soluciones para los artículos originales.

Palabras clave: Revisión. Métodos. Dominios científicos. Literatura.

Guide to systematic review of studies: an option for the methodology of Human Movement Sciences

Abstract: Gather knowledge is one of the objectives of the consultation literature. However, this is not restricted to this purpose. The objective of this test build practical guidance, whose thread can be followed by various areas of knowledge, resulting in studies with characteristics of repeatability and reproducibility. A systematic review question requires a clear, welldefined criteria and a conclusion that provides new information based on content panned. Thus, well structured reviews can assist in upgrading and construction of new guidelines for professional practice or going to the countryside in search of solutions for original articles.

Keywords: review. Methods. Scientific domains. Literature. 


\section{REFERÊNCIAS}

CASTRO, A. A. Revisão Sistemática e Meta-análise. In: GOLDENBERG, S.; GUIMARÃES, C. ALBERTO; CASTRO, A. A (Ed.) Elaboração e Apresentação de Comunicação Científica. São Paulo, 2009. p. 1-11. Disponível em:< http:// metodologia.org/wp-content/uploads/2010/08/meta1.PDF>. Acesso em: 30 jul. 2013.

CENTRE FOR REVIEWS AND DISSEMINATION (CRD). Finding studies for systematic reviews: A resource list for researchers. [Report]. York: University of York, out. 2012. Disponível em: <http://www.york.ac.uk/inst/crd/pdf/ Finding_studies_for_systematic_reviews.pdf>. Acesso em: 15 nov. 2013.

DE-LA-TORRE-UGARTE-GUANILO, M. C.; TAKAHASHI, R. F.;BERTOLOZZI, M. R. Revisão sistemática: noções gerais. Revista da Escola de Enfermagem USP, São Paulo, v. 45, n. 5, p. 1260 - 1266, out. 2011. doi: <http://dx.doi.org/10.1590/ S0080-62342011000500033>. Disponível em: <http://www.scielo.br/scielo.php? script=sci_arttext\&pid=S0080-62342011000500033\&lng=en\&nrm=iso<. Acesso em: 30 jul. 2013.

GONÇALVES, C. O.; CAMPANA, A. N.; TAVARES, M. C. Influência da atividade física na imagem corporal: uma revisão bibliográfica. Motricidade, Vila Real, v. 8, n. 2 , p. 70-82, 2012. <http://dx.doi.org/10.6063/motricidade.8(2).716>. Disponível em: <http://www.scielo.gpeari.mctes.pt/scielo.php?script=sci_arttext\&pid=S1646107X2012000200008\&lng=pt\&nrm=iso > . Acesso em 30 jul. 2013.

HOEFELMANN, C. P.; SANTOS, T. C.; MORETTI-PIRES, R. O. Revisões de artigos qualitativos por meta-síntese. In: SARAY, G.D.; MORETTI-PIRES, R. O. (Org.). Métodos e técnicas de Pesquisa Quantitativa Aplicada à Educação Física. Florianópolis: Tribo da Ilha, 2012. p. 149-155.

HIGGINS, J.; GREEN, S. (Ed.). Cochrane Handbook for Systematic Reviews of Interventions. Version 5.1.0 [updated March 2011]: The Cochrane Collaboration, 2011. Disponível em: <http://www.cochrane-handbook.org> . Acesso em: 12 maio 2013.

MEDINA, E. U.; PAILAQUILÉN, R. M. B. A revisão sistemática e a sua relação com a prática baseada na evidência em saúde. Revista Latino-Americana de Enfermagem, Ribeirão Preto, v. 18, n. 4, p. 1- 8, jul./ago. 2010. Disponível em: <http://www.scielo.br/pdf/rlae/v18n4/pt_23.pdf>. Acesso em: 10 nov. 2013.

MOHER, D.; LIBERATI, A.; TETZLAFF, J.; ALTMAN, D. G. A. Preferred Reporting Items for Systematic Reviews and Meta-Analyses: The PRISMA statement. Annals of Internal Medicine, Philadelphia v. 151, n.4, p. 264-269, jul. 2009. doi: 10.7326/ 0003-4819-151-4-200908180-00135. Disponível em: http://rds.epi-ucsf.org/ticr/ syllabus/courses/18/2012/03/29/Lecture/readings/PRISMA\%20Statement.pdf . Acesso em: 20 out. 2013. 
NOLL, M.; TARRAGÔ CANDOTTI, C.; VIEIRA, A. Escola postural: revisão sistemática dos programas desenvolvidos para escolares no Brasil. Movimento, Porto Alegre, v. 18, n. 4, p. 265-291, out./ dez. 2012. Disponível em: <http://www.redalyc.org/ articulo.oa?id=115324888016>. Acesso em: 20 nov. 2013.

RIERA, R.;ABREU, M. M.; CICONELLI, R. M. Revisões sistemáticas e metanálises na reumatologia. Revista Brasileira de Reumatologia, São Paulo, v. 46, n. 1, p. 811, jun. 2013. doi: http://dx.doi.org/10.1590/S0482-50042006000700003. Disponível em:< http://www.scielo.br/scielo.php?script=sci_arttext\&pid=S048250042006000700003\&lng=en\&nrm=iso >. Acesso em: 30 jul. 2013.

SAMPAIO, R. F.; MANCINI, M. C. Systematic review studies: a guide for careful synthesis of the scientific evidence. Revista Brasileira de Fisioterapia, São Carlos, v.11, n.1, p. 77-82, jan./fev. 2007. doi: http://dx.doi.org/10.1590/S141335552007000100013 . Disponível em: <http://www.scielo.br/scielo.php?pid=S141335552007000100013\&script=sci_arttext>. Acesso em: 13 nov. 2013.

SCHÜTZ, G. R.; SANT'ANA, A. S. S.; SANTOS, S. G. Política de periódicos nacionais em Educação Física para estudos de revisão sistemática. Revista Brasileira de Cineantropometria do Desempenho Humano, Santa Catarina, v. 13, n. 4, p. 313-319, 2011. doi: 10.5007/1980-0037.2011v13n4p313. Disponível em: <http:// www.scielo.br/pdf/rbcdh/v13n4/11.pdf>. Acesso em: 13 nov. 2013.

SEGURA-MUÑOZ, S. I.; TAKAYANAGUI, A. M. M.; SANTOS, C. B.; SANCHEZSWEATMAN, O. Revisão sistemática de literatura e metanálise: noções básicas sobre seu desenho, interpretação e aplicação na área da saúde. In: SIMPÓSIO BRASILEIRO DE COMUNICAÇÃ̃O EM ENFERMAGEM, 8, 2002, SIBRACEN, Ribeirão Preto (SP). Anais... Escola de Enfermagem de Ribeirão Preto da USP. Disponível em: $<$ http://www.proceedings.scielo.br/ scielo.php?script=sci_arttext\&pid=MSC0000000052002000200010\&lng=en\&nrm=abn> . Acesso em: 30 jul. 2013.

SOUZA, M. T.; SILVA, M. D. S.; CARVALHO, R. Revisão integrativa: o que é e como fazer. Einstein, São Paulo, v. 8, n. 1, p. 102-106, 2010. Disponível em: <http:// apps.einstein.br/revista/arquivos/PDF/1134-Einsteinv8n1_p102-106_port.pdf>. Acesso em: 23 jul. 2013.

SILVA, E. A. P. C.; SILVA, P. P. C.; SANTOS, A. R. M.; CARTAXO, H. G. O.; RECHIA, S.; FREITAS, C. M. S. M. Espaços públicos de lazer na promoção da qualidade de vida: uma revisão integrativa. Licere (Online), Belo Horizonte, v. 16, n. 2, jun., 1 - 18, 2013. Disponível em:<http://www.anima.eefd.ufrj.br/licere/pdf/ licereV16N02_a1.pdf >. Acesso em: 30 set. 2013.

SILVESTRIN, S.; SILVA, C. H.; HIRAKATA, V. N.; GOLDANI, A. A. S; SILVEIRA, P. P.; GOLDANI, M. Z. Grau de escolaridade materna e baixo peso ao nascer: uma metaanálise. Jornal de Pediatria, Rio de Janeiro, v. 89, n. 4, p. 339 - 345, jul./ago.2013. Disponível em: <http://jped.elsevier.es/pt/grau-escolaridade-materna-e-baixo/ articulo/90217724/>. Acesso em: 30 set. 2013.

THOMAS, J. R.; NELSON, J. K.; SILVERMAN, S. J. Métodos de pesquisa em atividade física. 6. ed. Porto Alegre: Artmed, 2012. 
Guia para estudos de revisão sistemática: uma opção...

VASCONCELOS, H. CristinaA.; FREITAS, R. W.J. F.; MARINHO, N. B. P.; DAMASCENO, M. M. C.; ARAÚJO, T. L.; LIMA, F. E. T. Eficácia de intervenções que utilizam o telefone como estratégia para o controle glicêmico: revisão integrativa da literatura. Texto \& Contexto Enfermagem, Florianópolis , v. 22, n. 1, , p. 239-246, jan./ mar 2013. Disponível em: < http://www.scielo.br/pdf/tce/v22n1/pt_29.pdf >. Acesso em: 30 set. 2013.

WRIGHT, R. W.;BRAND, R. A.; DUNN, W.; SPINDLER, K. P. How to write a systematic review. Clinical Orthopaedics and related research, New York: Springer, n. 455, p. 23-29, 2007. doi: 10.1097/BLO.0b013e31802c9098 . Disponível em: <http:/ /www.ncbi.nlm.nih.gov/pubmed/17279036>. Acesso em: 15 ago. 2013.

Este estudo conta com o apoio da CAPES na forma de concessão de bolsa de estudos de Mestrado.

Endereço para correspondência :

Isabelle Sena Gomes

Rua Professor Luis Soares, 170

CEP: $58300-320$

Centro,

Santa Rita, PB

Recebido em: 31.07 .2013

Aprovado em: 11.12.2013

Movimento, Porto Alegre, v. 20, n. 01, p. 395-411, jan/mar de 2014. 